\title{
Trombólise tardia e espontânea de artéria renal sem comprometimento funcional
}

\author{
Late and spontaneous thrombolysis of renal artery without \\ functional impairtment
}

\begin{abstract}
Autores
Claudio Pinho ${ }^{1}$

Henrique Pott Junior ${ }^{2}$

${ }^{1}$ Faculdade de Medicina, Centro Ciências da Vida, Pontifícia Universidade Católica de Campinas ${ }^{2}$ Faculdade de Medicina, Centro Ciências da Vida, Pontifícia Universidade Católica de Campinas
\end{abstract}

Data de submissão: 16/11/2008 Data de aprovação: 03/07/2009

Correspondência para: Henrique Pott Junior Rua São João Batista, 166 Cordeirópolis - SP CEP: $13490-000$

Tel: (19) 9243-9881

Declaramos a inexistência de conflitos de interesse.

\section{Resumo}

A aterosclerose em artérias renais é um importante fator desencadeante de tromboses com subsequente comprometimento da função e da viabilidade renal. A oclusão aguda das artérias renais por trombo ou êmbolo é causa incomum e potencialmente reversível de falência renal. Todavia, a duração e o grau de oclusão arterial compatível com a manutenção da viabilidade do parênquima renal ainda não estão bem estabelecidos, razão pela qual o diagnóstico precoce e a intervenção são importantes. O objetivo deste artigo é descrever um caso de trombose de artéria renal de rim funcional único, com lise espontânea e tardia do trombo seguida de recuperação funcional inesperada.

Palavras-chave: aterosclerose, artéria renal, trombose, circulação colateral.

[J Bras Nefrol 2009;31(2):228-231]@Elsevier Editora Ltda.

\section{INTRODUÇÃo}

A oclusão aguda das artérias renais por trombo ou êmbolo é causa incomum e potencialmente reversível de falência renal. ${ }^{1,2}$ O tempo e o grau de oclusão arterial compatíveis com a manutenção da viabilidade do parênquima renal ainda não estão bem estabelecidos, o que realça a grande importância do diagnóstico precoce; se o rim não estiver amplamente infartado, a revascularização pode levar ao retorno espontâneo da função renal, pois as lesões isquêmicas afetam preferencialmente o epitélio tubular, que tem propriedades regenerativas. No entanto, a recuperação funcional do rim fica comprometida quanto maior for o período de isquemia. ${ }^{1,2}$

\section{Abstract}

Renal artery atherosclerosis is an important triggering factor for thrombosis followed by impairment of renal function and viability. The acute occlusion of the renal artery by a thrombus or an embolus is an unusual and potentially reversible cause of renal failure. However, the duration and degree of arterial occlusion compatible with the viability of the renal parenchyma have not been well established, hence the importance of early diagnosis and intervention. The purpose of this study is to describe a case of renal artery thrombosis of a functionally single kidney, with late and spontaneous thrombolysis followed by unexpected functional recovery.

Keywords: atherosclerosis, renal artery, thrombosis, collateral circulation.

A aterosclerose é um importante fator desencadeante de tromboses. A natureza crônica do processo de estenose de artéria renal pela aterosclerose possibilita o desenvolvimento de vias alternativas de circulação, o que permite irrigação suficiente para manter a viabilidade renal em situações de obstrução aguda da artéria renal. ${ }^{3,4}$

A seguir, relatamos um caso de trombose de artéria renal de rim funcional único, sem comprometimento do parênquima renal e com retorno tardio espontâneo da função renal.

\section{Relato de Caso}

Paciente de sexo masculino, 53 anos, branco, foi admitido em quadro abrupto de dor abdominal intensa, em fossa 
ilíaca e flanco esquerdo, acompanhada de vômitos e anúria. Histórico de tabagismo de um maço/dia por cerca de 24 anos, etilismo, padrão alimentar abusivo de sal e gorduras, sedentário, com estresse elevado e em acompanhamento ambulatorial com história de hipertensão arterial sistêmica (HAS) detectada em exame periódico aos 42 anos.

A história pregressa revelava que, aos 49 anos, apresentou crise hipertensiva com necessidade de internação e, após alta hospitalar, manteve níveis de pressão arterial $200 \times 106 \mathrm{mmHg}$, apesar do uso de clortalidona $50 \mathrm{mg}$, captopril $50 \mathrm{mg} / \mathrm{dia}$ e nifedipina $20 \mathrm{mg} / \mathrm{dia}$. A angiografia de aorta abdominal realizada aos 49 anos demonstrou oclusão total desse vaso após troncos viscerais, artéria renal esquerda pérvia e artéria renal direita ocluída, artéria mesentérica superior e tronco celíaco pérvios e artérias femorais contrastando-se tardiamente. A tomografia computadorizada de abdômen confirmou os achados citados e acrescentou redução abrupta do calibre vascular aórtico após artéria renal esquerda, com grande quantidade de ateromas alcançando até as artérias ilíacas. A ecografia abdominal demonstrou atrofia renal direita e rim esquerdo vicariante. $\mathrm{O}$ ecocardiograma mostrava estruturas cardíacas dentro dos parâmetros de normalidade. A Monitorização Ambulatorial da Pressão Arterial (MAPA) aos 49 anos mostrava controle pressórico com médias de 132 x $84 \mathrm{mmHg}$ nas 24 horas, sendo, na vigília, de 131 × $84 \mathrm{mmHg}$ e, no sono, de 133 × $88 \mathrm{mmHg}$, com ausência de descenso fisiológico. A partir desse episódio de crise hipertensiva, apresentou adesão ao tratamento e controle dos níveis pressóricos.

O exame físico de admissão, aos 53 anos, revelou paciente desidratado, descorado, frequência cardíaca (FC) $96 \mathrm{bpm}$, pressão arterial de 150 x $90 \mathrm{mmHg}$, e, à ausculta abdominal, sopro rude $+3 /+4$ em região epigástrica. A história do paciente e o exame físico direcionaram a investigação clínica da insuficiência renal aguda para uma causa vascular. Nessa ocasião, os exames laboratoriais demonstraram hemoglobina $12,2 \mathrm{~g} / \mathrm{dL}$; hematócrito $36,2 \%$; creatinina $3,9 \mathrm{mg} / \mathrm{dL}$; ureia $78,0 \mathrm{mg} / \mathrm{dL}$; sódio $130 \mathrm{mEq} / \mathrm{L}$; e potássio 4,6 mEq/L. A ultrassonografia de loja renal revelou trombose de artéria renal esquerda. Como a história revelava sintomas de mais de 48 horas, foi contraindicado tratamento cirúrgico (revascularização renal esquerda). A evolução dos dados laboratoriais de creatinina e ureia durante a internação hospitalar pode ser observada no Gráfico 1. A ecografia abdominal realizada sete dias após o início dos sintomas mostrou rim esquerdo com dimensões normais (limite su- perior), fluxo arterial e venoso presentes e estenose na origem da artéria renal esquerda. Rim direito atrófico e ausência de fluxo na artéria renal direita.

Gráfico 1. Evolução dos dados laboratoriais de creatinina $(\mathrm{mg} / \mathrm{dL})$ e ureia $(\mathrm{mg} / \mathrm{dL})$ durante os seis dias de internação e após dois meses de alta hospitalar.

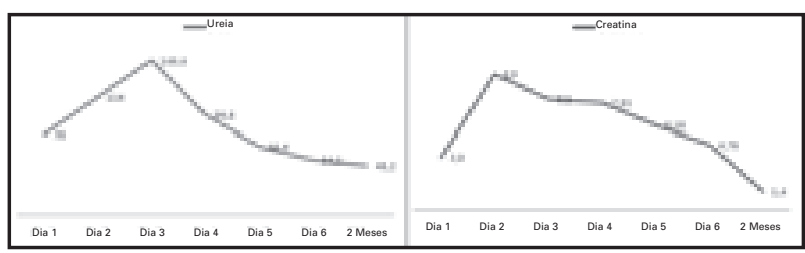

Dois meses após o quadro de dor aguda, realizouse renograma com estudo radioisotópico utilizando DTPA-tecnécio-99m para avaliação da função renal, que demonstrou aortopatia aterosclerótica com comprometimento de artéria renal esquerda, função glomerular de rim esquerdo diminuída de grau moderado e exclusão funcional de rim direito.

Nessa ocasião, os exames laboratoriais demonstraram hemoglobina 14,3 g/dL; hematócrito 43,2\%; creatinina $1,4 \mathrm{mg} / \mathrm{dL}$; clearance de creatinina $109 \mathrm{~mL} /$ min; potássio 4,0 mEq/L; colesterol total $221 \mathrm{mg} / \mathrm{dL}$; LDL-colesterol $136 \mathrm{mg} / \mathrm{dL}$; HDL-colesterol $47 \mathrm{mg} /$ dL; e triglicérides $136 \mathrm{mg} / \mathrm{dL}$.

Três meses após internação, o paciente foi submetido com sucesso a angioplastia e colocação de stent em artéria renal esquerda, com o objetivo de prevenir nefropatia isquêmica, uma vez que a função renal fora recuperada e a pressão arterial estava bem controlada.

$\mathrm{Na}$ evolução pós-angioplastia transluminal, o paciente estava assintomático, exceto por queixa de impotência, e, após reabilitação cardiovascular, não apresentou claudicação durante as caminhadas. Em uso de furosemida $80 \mathrm{mg} / \mathrm{dia}$, losartan $50 \mathrm{mg} / \mathrm{dia}$, propranolol $120 \mathrm{mg} / \mathrm{dia}$, nifedipina oros $60 \mathrm{mg} / \mathrm{dia}$, sinvastatina $20 \mathrm{mg} / \mathrm{dia}$, ácido acetilsalicílico $100 \mathrm{mg} /$ dia, alopurinol $300 \mathrm{mg} / \mathrm{dia}$ e omeprazol $20 \mathrm{mg} / \mathrm{dia}$. Exames laboratoriais sete anos após o evento: colesterol total, $157 \mathrm{mg} / \mathrm{dL}$; LDL-colesterol, $78 \mathrm{mg} / \mathrm{dL}$; HDL-colesterol, $46 \mathrm{mg} / \mathrm{dL}$; triglicérides, $167 \mathrm{mg} / \mathrm{dL}$; potássio, 4,2 mEq/L; ureia, $51 \mathrm{mg} / \mathrm{dL}$; creatinina, 1,4 $\mathrm{mg} / \mathrm{dL}$; clearance de creatinina, $71 \mathrm{~mL} / \mathrm{min} / 1,73 \mathrm{~m}^{2}$; proteinúria, 1,6 g/24h; eletrocardiograma e ecocardiograma aos 60 anos se mantiveram sem alterações. MAPA aos 61 anos: média 24h, 118 x $74 \mathrm{mmHg}$, na vigília, 118 x 77 e sono 114 x $64 \mathrm{mmHg}$. 
Figura 1. Angiografia de aorta: oclusão total de aorta após troncos viscerais (seta 1), artéria renal esquerda pérvia (seta 2) e artéria renal direita ocluída, artéria mesentérica superior e tronco celíaco pérvios.

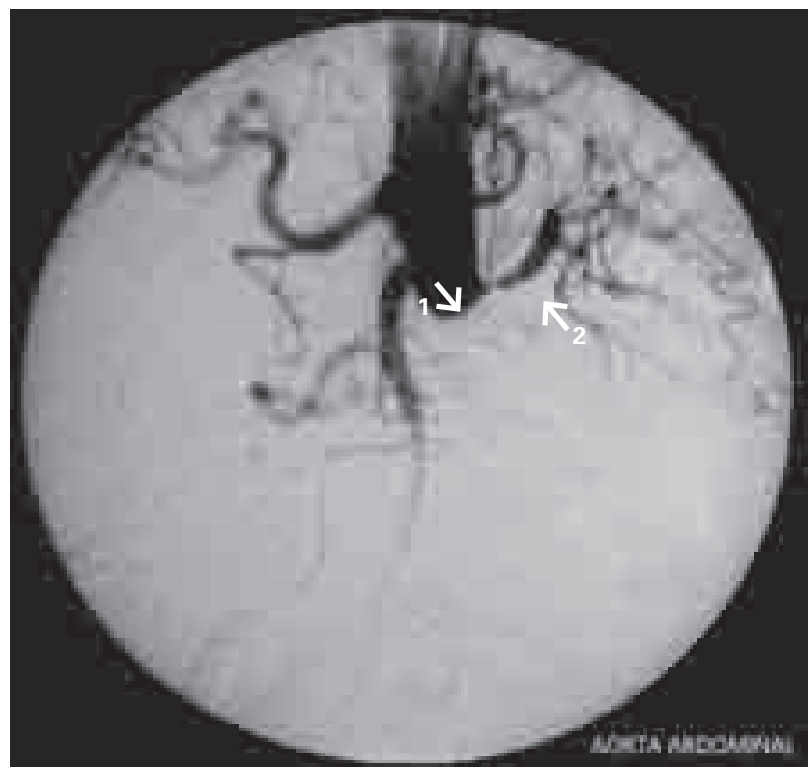

\section{Discussão e Conclusões}

As doenças cardiovasculares são a principal causa de morte em nosso país, atingindo cerca de $30 \%$ dos óbitos em todas as faixas etárias. ${ }^{1-3}$ Os estudos Framingham, MRFIT e PROCAM demonstram o indiscutível papel das dislipidemias (LDL-coleterol elevado e HDL-coleterol diminuídos), hipertensão arterial sistêmica, fumo, idade e diabetes mellitus como fatores de risco independentes para aterosclerose. Outros fatores de risco causam menos impacto no risco. São eles: história familiar precoce de doença isquêmica do coração, obesidade, etnia e fatores psicossociais.

A aterosclerose é uma doença sistêmica responsável por $90 \%$ dos casos de estenose da artéria renal (EAR). ${ }^{5} \mathrm{~A}$ estenose aterosclerótica da artéria renal (EAAR) é a doença primária mais comum das artérias renais e resulta em hipertensão renovascular e nefropatia isquêmica. ${ }^{6}$ Ademais, a estenose da artéria renal está associada a eventos cardiovasculares (infarto do miocárdio, acidente vascular encefálico) e morte por causa cardiovascular. ${ }^{6}$

Não obstante, a oclusão aguda completa ou quase completa tanto da artéria renal principal quanto de um grande ramo intrarrenal pode ocorrer em razão do desenvolvimento agudo de trombose desses vasos ou por fenômenos embólicos. ${ }^{4}$ Nesses casos, o processo etiológico desempenha papel decisivo sobre o impacto da isquemia no parênquima renal. ${ }^{7,8}$
Figura 2. Tomografia abdominal: redução abrupta do calibre vascular após artéria renal esquerda com acentuação dos ateromas (seta).

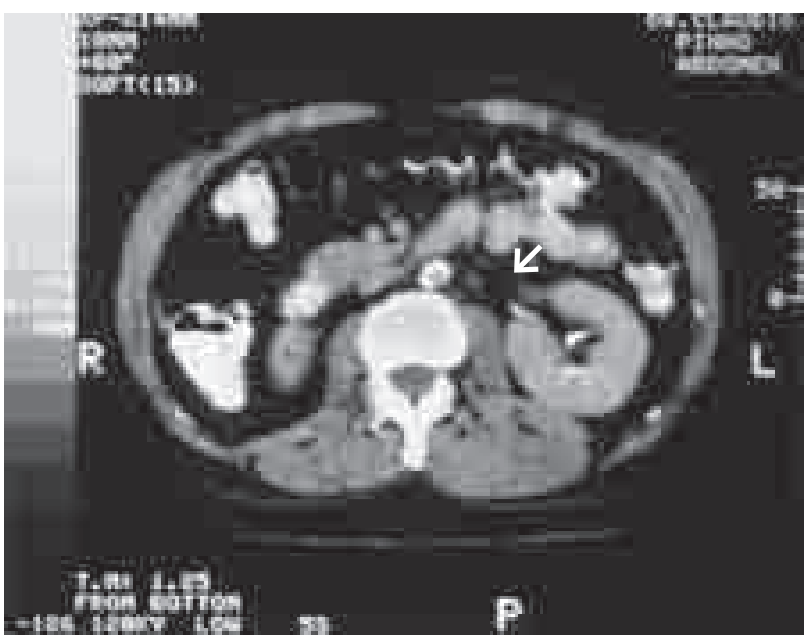

Os rins recebem cerca de $20 \%$ do débito cardíaco, o que torna os fenômenos embólicos das pequenas artérias intrarrenais relativamente comuns, enquanto a trombose necessita de lesão prévia, como ruptura de placa de ateroma ou vasculite. Atualmente, sabese que aterosclerose é causa potencialmente reversível de oclusão aguda de artéria renal por tromboses, relacionadas ao fluxo vascular anormal e à perda de integridade endotelial. ${ }^{3,4}$

A duração e o grau de oclusão arterial compatíveis com a manutenção da viabilidade do parênquima renal ainda não estão bem estabelecidos, razão pela qual o diagnóstico e o tratamento precoce são importantes. Em modelos experimentais com ratos, o clampeamento e a ligadura da artéria renal demonstraram que o rim não tolera ausência de circulação com duração superior a 90 minutos. Frise-se que, acima de $50 \mathrm{mi}-$ nutos, as sequelas já são parcialmente irreversíveis. ${ }^{8,9}$ Mesmo a oclusão total de uma das artérias renais principais não consegue causar elevação nos níveis de ureia e creatinina séricos, caso o rim contralateral esteja em perfeito funcionamento. A oclusão aguda só entra no diagnóstico diferencial de insuficiência renal aguda quando é bilateral ou quando o rim contralateral é mal funcionante ou não funcionante, como no caso relatado.

Quando uma artéria renal principal se oclui repentinamente, embora comprometa a função renal, a viabilidade do rim pode ser mantida por circulação colateral preexistente. Em tais casos, pressupõe-se a existência prévia de oclusão arterial parcial que te- 
nha permitido o desenvolvimento de circulação colateral suficiente para nutrir o parênquima, mas não para manter a função renal. Dessa forma, a presença da neovascularização pode impedir lesão celular definitiva, deixando as células isquêmicas em estado de hibernação e, com isso, obtendo recuperação funcional se a circulação for restabelecida, como ocorreu de maneira espontânea no caso relatado. Heider et al. demonstraram que o baixo fluxo sanguíneo renal por baixa pressão arterial (inferior a $30 \mathrm{mmHg}$ ), que é inadequada para manter filtração glomerular e função renal mínima, ainda pode ser adequado para fornecer oxigenação, visando à sobrevivência do órgão. ${ }^{3,8}$ Essa circulação colateral se torna funcionalmente importante após oito horas do início do evento oclusivo e ainda pode ser mais efetiva em rins com estenose arterial preexistente. ${ }^{12}$ Yune e Klatte demonstraram, por meio de arteriografia, a existência de circulação colateral em $40 \%$ dos pacientes com estenose de artéria renal. ${ }^{13}$

Frente a um quadro de insuficiência renal aguda devido à oclusão da artéria renal, a escolha do tratamento ideal deve ser individualizada, em vista da duração e do grau de oclusão arterial. Pacientes com oclusão unilateral devem ser tratados de forma conservadora, com observação e anticoagulação, pois, frequentemente, há recanalização. Quanto à terapia nos casos de oclusão bilateral ou oclusão de um rim único, atualmente não há acordo com relação ao melhor tratamento, uma vez que é difícil avaliar as intervenções terapêuticas relatadas, pois a maioria dos estudos é retrospectiva e não existem análises controladas comparativas entre intervenção cirúrgica e uso de fibrinolítico. Embora alguns autores tenham sugerido que o tratamento fibrinolítico deve ser utilizado como primeira escolha no tratamento de todos os casos de insuficiência renal por tromboembolia, não há consenso quanto às indicações ou à duração dessa terapia. ${ }^{14}$ A angioplastia transluminal percutânea também se provou eficaz em pacientes selecionados. ${ }^{15}$

Por conseguinte, a natureza crônica do processo de estenose de artéria renal pela aterosclerose possibilita o desenvolvimento de circulação colateral, que, por sua vez, permite irrigação suficiente para manter a viabilidade renal em caso de obstrução aguda da artéria renal. ${ }^{4,7,10}$

Dessa forma, se o rim não estiver amplamente infartado, pode haver retorno espontâneo da função renal, porque as lesões isquêmicas afetam preferencialmente o epitélio tubular, que tem propriedades regenerativas. No entanto, o retorno espontâneo da função renal como nesse caso não é esperada. ${ }^{4}$ Por conseguinte, assim como em outros casos descritos na literatura, a existência de longo intervalo de tempo entre a trombose da artéria renal com anúria e a avaliação e a intervenção médica talvez não prediga infarto renal.

\section{REFERÊNCIAS}

1. Dardik A, Ballermann BJ, Williams GM. Successful Delayed Bilateral Renal Revascularization during Active Phase of Takayasu's arteritis. J Vasc Surg 1998; 27:552-4.

2. Heider CF, Morris GC Jr, Moyer JH. The Protective Effect of Subfiltration Arterial Pressure on the Kidney. Surg Forum 1956; 6:623-7.

3. DataSUS. Disponível em: <http://www.datasus.gov. br>. Acesso em: 01 outubro 2008.

4. Lacombe M. Acute Non-Traumatic Obstructions of the Renal Artery. J Cardiovasc Surg (Torino) 1992; 33:163-8.

5. Textor SC. Ischemic Nephropathy: Where are We Now? J Am Soc Nephrol 2004; 15:1974-82.

6. Kendrick J, Chonchol M. Renal Artery Stenosis and Chronic Ischemic Nephropathy: Epidemiology and Diagnosis. Adv Chronic Kidney Dis 2008; 15:355-62.

7. Nordmann AJ, Woo K, Parkes R, Logan AG. Balloon Angioplasty or Medical Therapy for Hypertensive Patients with Atherosclerotic Renal Artery Stenosis? A Meta-Analysis of Randomized Controlled Trials. Am J Med 2003;114:44-50.

8. Mesnard L, Delahousse M, Raynaud A et al. Delayed Angioplasty After Renal Thrombosis. Am J Kidney Dis 2003;41:E9-12.

9. Handley C, Heider C, Morris GC Jr, Moyer JH. Renal Failure. I. The Effect of Complete Renal Artery Occlusion for Variable Periods of Time as Compared to Exposure to Sub-Filtration Arterial Pressures Below 30mm Hg for Similar Periods. Ann Surg 1957; 145:41-58.

10. van Damme H, Rorive G, Limet R. Reversal of Acute Renal Failure by Kidney Revascularisation. Eur J Vasc Endovasc Surg 1996; 11:134-9.

11. Cuppage FE, Neagoy DR, Tate A. Repair of the Nephron Following Temporary Occlusion of the Renal Pedicle. Lab Invest 1967; 17:660-74.

12. Love L, Bush IM. Early Demonstration of Renal Collateral Arterial Suppl. Am J Roentgenol Radium Ther Nucl Med 1968; 104:296-301.

13. Yune HY, Klatte EC. Collateral Circulation to an Ischemic Kidney. Radiology 1976; 119:539-46.

14. Lessman RK, Johnson SF, Coburn JW, Kaufman JJ. Renal Artery Embolism: Clinical Features and Long-Term Follow-Up of 17 cases. Ann Intern Med 1978; 89:477-82.

15. White CJ. Catheter-Based Therapy for Atherosclerotic Renal Artery Stenosis. Circulation 2006;113:1464-73. 